\title{
XEROGENIC POTENCY OF CHRONIC USAGE OF TRAMADOL IS RELATED TO STRUCTURAL CHANGES IN THE PAROTID GLAND
}

\author{
Menatalla Mohamed Elhindawy * and Fawzy Abo yhya Ali **
}

\begin{abstract}
Objectives: This study was designed to detect weather the xerogenic potency of chronic consumption of tramadol is related to its neural effect only or it is also related to structural changes of the parotid gland.

Methods: 16 adult male albino rats weighing (200-250 gm) were divided into 3 groups; control group ( 4 rats) received distilled water, tramadol group ( 6 rats) received $40 \mathrm{mg} \mathrm{kg}$ of tramadol for 20 and 30 days, and tramadol withdrawal group ( 6 rats) received $40 \mathrm{mg} \mathrm{kg}$ of tramadol then tramadol was withdrawn for 20 and 30 days. Parotid gland was processed for $\mathrm{H} \& \mathrm{E}$, toluidine blue stain and capsase-3 immuno-histochemical stain, also levels of malondialdehyde (MDA) were detected.
\end{abstract}

Results: H\&E sections showed disorganization and apoptosis in tramadol treated groups; after tramadol withdrawal the glands restored its normal architecture, mitotic and apoptotic activity were observed. Apoptotic findings were confirmed with the results of caspase-3 immuno-histochemical staining. Toluidine blue stain detected decreased amount of zymogen granules within the acinar cells of tramadol group which increased gradually after its withdrawal. Levels of MDA were increased in tramadol groups indicating increased level of oxidative stresses, after tramadol withdrawal MDA levels decreased again.

Conclusion: Xerogenic potency of tramadol could be attributed to structural changes within the gland not only to its neural effect.

KEY WORDS: Tramadol; xerogenic potency; caspase- 3; oxidative stress

\section{INTRODUCTION}

Salivary secretion is entirely mediated by nervous mechanisms, para-sympathetic and sympathetic nerves are in contact with acinar, ductal and myoepithelial cells and blood vessels. ${ }^{1}$
Salivary hypofunction or xerostomia is induced by suppression of impulse traffic from the salivary nuclei to salivary glands. ${ }^{2}$ it is most frequently secondary to medication consumption. Medicationinduced salivary hypofunction, is associated with

* Associate Professor of Oral Biology, Oral Biology Department, Faculty of Dentistry, Mansoura University, Egypt ** Associate Professor of Oral Biology, Oral biology Department, Faculty of Dentistry, Zagazig University, Egypt 
blocking of anticholinergic muscarinic (M3) receptor for treatment of irritable bladders, or activation of alpha-2-adrenergic receptors in the central nervous system, also antidepressants can cause salivary hypofunction. ${ }^{3}$

Tramadol is a synthetic analogue of codeine which acts through both opioid and non-opioid mechanisms of action. ${ }^{4,5}$ It inhibits the ascending pain path way altering the perception and response to pain thought binding to $\mu$-opiate receptors in the central nervous system, it also inhibits the reuptake of norepinephrine and serotonin, which also modifies the ascending pain path ways. ${ }^{6}$

Oral tramadol was shown to reduce salivary flow by $75 \%$ in a clinical study on healthy volunteers. ${ }^{7}$ In a randomized, double blind and placebo-controlled trail on volunteered students; administration of tramadol (50 mg 3/day over two days), the salivary flow was reduced by $64 \%$ and protein output by $52 \% .^{8}$

In brain tissue; tramadol administration induces oxidative stress and a significant decrease in the antioxidants in rat model. ${ }^{9}$ Elevated levels of Malondialdehyde (MDA) is considered a useful measure of oxidative stress status as it indicates an increase of free radical generation. ${ }^{10}$

Oxidative stress is defined as a steady-state of reactive oxygen species (ROS) is transiently or chronically enhanced, disturbing cellular metabolism and its regulation and damaging cellular macromolecules. ${ }^{11}$ The target of ROS damage includes all groups of biomolecules which if combined with weakening of antioxidant systems may result in permanent changes in the redox state of DNA, RNA, proteins, lipids, and carbohydrates and leads to the loss of the biological function of the cells. ${ }^{12}$

The current study was designed to investigate the possible structural damage occurs in the parotid gland after chronic consumption of tramadol and its effect on salivary secretion.

\section{MATERIALS AND METHODS}

\section{Animals}

- 16 adult Albino rats weighting (200-250 g) were used in this experiment. The animals were accommodated for at least two weeks in individual cage and received standard food for rodents before starting the experiment.

- Rats were divided into 3 groups

I. Control group.

4 rats, received distilled water via gastric tube daily, one rat was sacrificed with each scarification period of other groups.

II. Tramadol group.

6 rats, received $40 \mathrm{mg} \backslash \mathrm{kg}{ }^{13}$ via gastric tube daily and were divided as follow

a) 3 rats received the drug for 20 days and then sacrificed.

b) 3 rats received the drug for 30 days and then sacrificed.

III. Tramadol withdrawal group.

6 rats, received $40 \mathrm{mgkg}{ }^{13}$ via gastric tube daily for 30 days and were divided as follow

a) 3 rats received distilled water for 20 days after tramadol withdrawal and then sacrificed.

b) 3 rats received distilled water for 30 days after tramadol withdrawal and then sacrificed.

\section{Histopathological examination.}

- Specimens from parotid gland were taken from each group and were fixed in $10 \%$ neutral buffer formalin, embedded in paraffin, sectioned at 5 microns and prepared for Hematoxylin and Eo$\sin (H \& E)$ stain and Toluidine blue stain.

\section{Immucohistochemistry examination.}

Sections were immune-stained using avidinbiotin technique. Sections were stained with rabbit 
anti-rat caspase-3 (Sigma- Aldrich, St Louis, USA). Slides were digitized using Olympus ${ }^{\circledR}$ digital camera installed on Olympus ${ }^{\circledast}$ microscope. The resulted images were analyzed on Intel ${ }^{\circledR}$ Core $\mathrm{I} 3{ }^{\circledR}$ based computer using VideoTest Morphology ${ }^{\circledR}$ software (Russia) with a specific built-in routine for immunostain quantification.

Paraffin-embedded sections were heated to $60{ }^{\circ} \mathrm{C}$ to melt the paraffin, then washed in xylene and rehydrated by graded washing in $100,95,80,70$, $50 \%$ ethanol in distilled water (each concentration for $20 \mathrm{~min}$ ). Sections were then incubated in $0.3 \%$ Triton $\mathrm{X}-100$ at $37{ }^{\circ} \mathrm{C}$ for $30 \mathrm{~min}$, and, for antigen retrieval, placed in a boiling water bath for $15 \mathrm{~min}$. They were next placed in $0.3 \%$ hydrogen peroxide for $15 \mathrm{~min}$. The sections were washed with 0.01 $\mathrm{mol} / \mathrm{l}$ phosphate-buffered saline (PBS; $\mathrm{pH} 7.4,4{ }^{\circ} \mathrm{C}$ ) $(3 \times 5 \mathrm{~min})$ between each step. Sections were then incubated in biotinylated goat anti-rabbit IgG serum (Beijing Zhongshan Goldenbridge Biotechnology Co., Ltd. Beijing, China) for $2 \mathrm{~h}$, and then with rabbit anti-rat caspase-3 polyclonal antibody (1:100 dilution in $0.01 \mathrm{~mol} / \mathrm{l} \mathrm{PBS}$; Beijing Zhongshan Golden Bridge Biotechnology Co, Ltd. Beijing, China) at room temperature for $1 \mathrm{~h}$ and then at $4{ }^{\circ} \mathrm{C}$ overnight. The following day, the sections were placed at room temperature for $1 \mathrm{~h}$, and then avidin- biotin complex (ABC) staining kit (Beijing Zhongshan Golden Bridge Biotechnology Co. Ltd. Beijing, China) was used. Finally, each section was stained with DAB, using a staining kit (Beijing Zhongshan Golden Bridge Biotechnology Co. Ltd. Beijing, China), for approximately $5 \mathrm{~min}$, and the immunoreactive products were visualized as a brown stain. PBS $(0.01 \mathrm{~mol} / \mathrm{l})$ was used for washing between each step $(3 \times 5 \mathrm{~min})$. Then, hematoxylin was used to stain the cell nuclear in order to facilitate observation. After dehydration and mounting of neutral resins, sections were observed under a light microscope (Leica; BX51). The same process was used for negative controls, except that $0.01 \mathrm{~mol} / \mathrm{l}$ PBS was used instead of rabbit anti-rat caspase-3 polyclonal antibodies.

\section{Malondialdehyde level detection.}

- Parotid gland samples were homogenized in 5-10 $\mathrm{ml}$ cold buffer (i.e. $50 \mathrm{mM}$ potassium phosphate, $\mathrm{pH}$ 7.5. $1 \mathrm{mM}$ EDTA). Homogenates were centrifuged at $10000 \times \mathrm{g}$ for 15 minutes at $4^{\circ} \mathrm{C}$ and the supernatant was kept at $-80^{\circ} \mathrm{C}$ till used for analysis of lipid peroxides (malondialdehyde, MDA) which were analyzed using colorimetric kit (Sigma- Aldrich, St Louis, USA) The value is expressed as nmol/g tissue.

\section{Statistical analysis}

- All data were analyzed using SPSS17.0 and recorded as means \pm standard deviations "mean \pm SD”. Caspase 3 expression and MDA levels were analyzed using two-way ANOVA test.

\section{RESULTS}

\section{Histopathological results.}

\section{Control group}

Examination of H\&E sections of parotid gland of all control group samples showed normal architecture of serous acini with pyramidal cells and rounded basal nucleus. Toluidine blue stain revealed dark blue zymogen granules within the cytoplasm and dark blue basal nuclei.

\section{Tramadol group}

Sections stained with H\&E after 20 days of tramadol administration showed loss of the basal position of the nuclei of acinar cell as well as signs of apoptosis "pyknosis and chromatin condensation", and the cytoplasm showed numerous vacuoles "fig.1 A". Toluidine blue section showed faintly blue stained vacuolated cytoplasm with dark stained nuclei "fig. $2 \mathrm{~A}$ ".

After 30 days of tramadol administration; H\&E sections showed massive destruction of acinar architecture marked by disorganization of the acinar cells and its nuclei, increased number of acinar cells 
showing signs of apoptosis and vacuolization of cytoplasm"fig.1 B". Sections stained with toluidine blue showed depleted acini with decreased amount of zymogen granules within the cytoplasm also marked vacuolated cytoplasm "fig. 2 B".

\section{Tramadol withdrawal group}

H\&E section after 20 days of tramadol withdrawal showed that acinar cells regained their basely located nucleus with reduced vacuoles in their cytoplasm, apoptotic findings were also reduced "fig.1 C". Toluidine blue sections revealed less vacuolated acinar cells cytoplasm with few zymogen granules at the apical portion when compared to tramadol treated groups "fig. $2 \mathrm{C}$ ".

After 30 days of withdrawal; H\&E section showed acinar cell returned almost to its normal architecture, mitotic activity as well as apoptotic findings were still observed "fig.1 D". Toluidine blue sections although some acinar cells showed vacuolated cytoplasm but acinar cells showed dark blue stained basal nuclei and moderate amount of zymogen granules within the cytoplasm "fig. 2 D".

\section{Immunohistochemistry results}

Brown color representing positive staining could be observed in the cytoplasm of acinar cells.

\section{Control group}

Control sections stained of the parotid gland at all time periods showed expression of caspase- 3 with mean percentage of $(86.3 \pm 1.4 \& 87.3 \pm 1.4)$.

\section{Tramadol treated group}

Section stained with anti-caspase 3 expressed more positive cells for caspase 3 activity after 30 days of tramadol administration with mean percentage of $(149.7 \pm 3.9)$ "fig.3 B" when compared to acinar cell expression after 20 days with mean percentage of (126.03 \pm 3.6$)$ "fig. 3 A" "fig.4". Statistical analysis showed significant difference between caspase- 3 activities between both tramadol treated groups $(\mathrm{p}<0.05)$

\section{Tramadol withdrawal group}

Sections stained with anti-caspase 3 after 30 days of tramadol withdrawal showed the least expression of the caspase -3 activity among all groups with mean percentage of $(112.49 \pm 3.3)$ "fig.3 D"

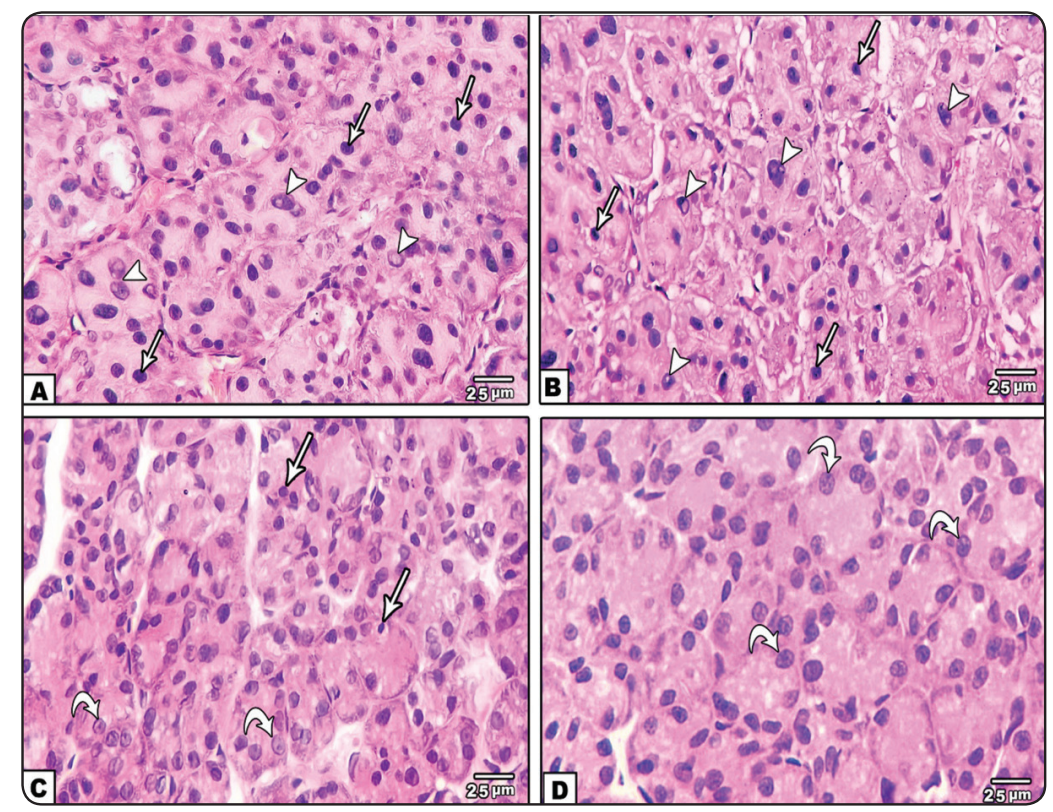

Fig 1: photomicrograph of parotid gland, (A) tramadol treated group for 20 days shows loss of acinar organization, acinar cells showed apoptotic signs as pyknosis (white arrow) and chromatin condensation (arrow heads)(B) tramadol treated group for 30 days shows disorganized acini with marked vacuolization of cytoplasm and more apoptotic signs (C) tramadol withdrawal group for 20 days shows less vacuolated cytoplasm with some zymogen granules within the acinar cells (D) tramadol withdrawal group for 30 days shows moderate amount of zymogen granules within the acinar cells. (H\&E X 400) 
while sections after 20 days of tramadol withdrawal showed caspase expression of mean percentage of

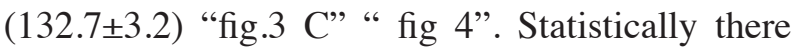
were a significant difference between both tramadol withdrawal groups as well as between tramadol withdrawal group and tramadol treated group $(\mathrm{p}<0.05)$.

\section{Malondialdehyde level detection results}

Malondialdehyde levels of parotid extract in control group showed $(23.1 \pm 5.1 \& 27.9 \pm 5.4)$ these levels increased in tramadol treated group for 20 days to reach $(123.1 \pm 24.7)$ and continued to increase to reach $(132.9 \pm 38.3)$ at the treated group for 30 days. Levels of MDA decreased to reach $(44.5 \pm 11.2)$ after 20 days of tramadol withdrawal and continued to reduce to reach $(38.8 \pm 12.9)$ after 30 days of withdrawal. There was statistical significance between tramadol treated group and tramadol withdrawal group in MDA levels $(\mathrm{p}<0.05)$.
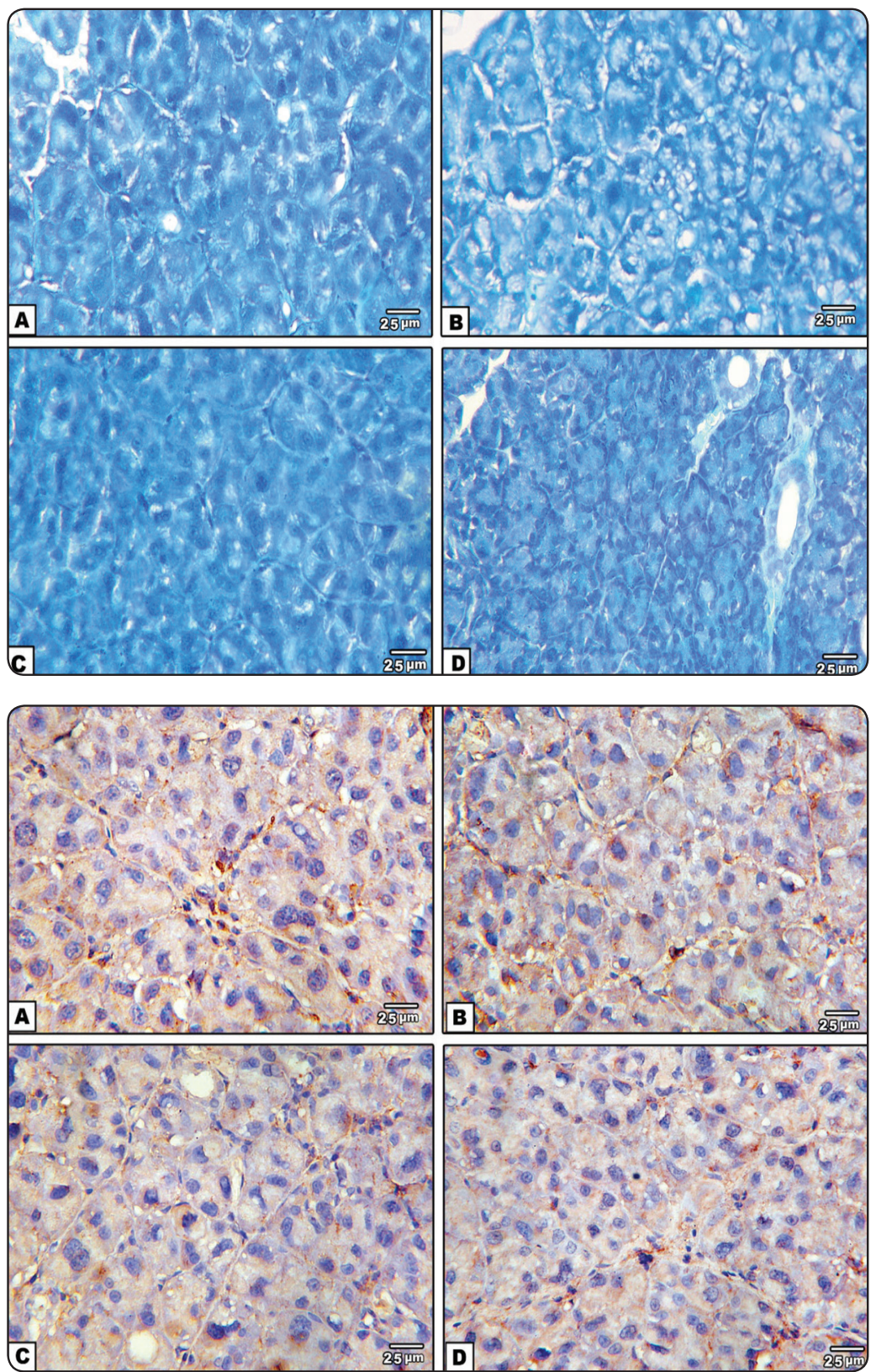

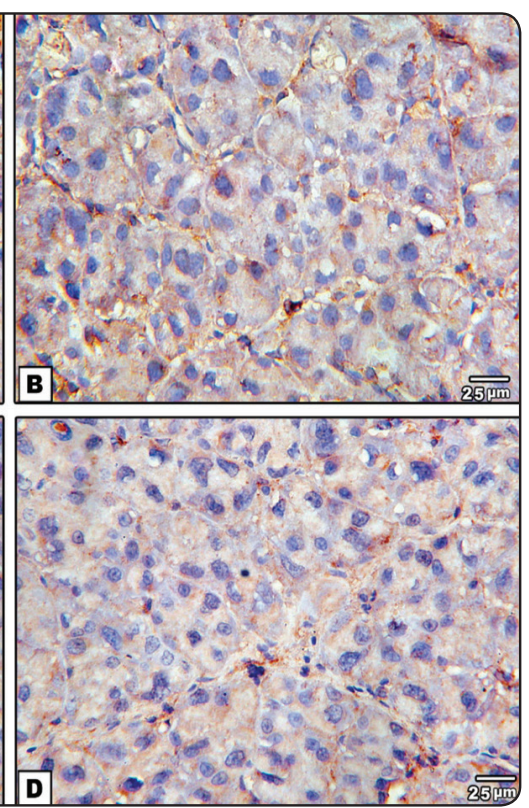

Fig (2) Photomicrograph of parotid gland, (A) tramadol treated group for 20 days shows faint blue vacuolated cytoplasm (B) tramadol treated group for 30 days shows depleted disorganized acini with marked vacuolization of cytoplasm (C) tramadol withdrawal group for 20 days shows less vacuolated cytoplasm with some zymogen granules within the acinar cells (D) tramadol withdrawal group for 30 days shows moderate amount of zymogen granules within the acinar cells. (Toluidine blue X 400)

Fig. (3) Photomicrograph of parotid gland showing anti capase-3 activity (A) tramadol treated group for 20 days (B) tramadol treated group for 30 days (C) tramadol withdrawal group for 20 (D) tramadol withdrawal group for 30 days (IHC caspase 3, X 400) 


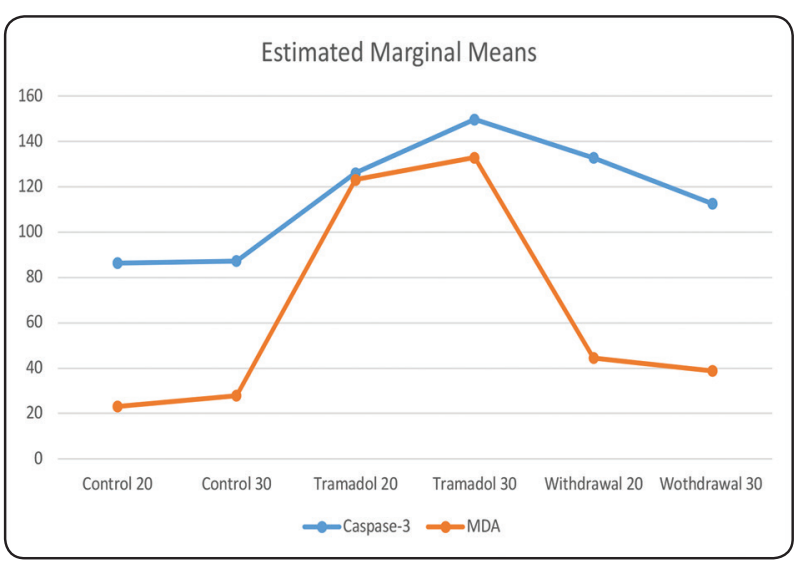

Fig. (4) Estimated marginal means for both Caspase 3 and MDA expression among different groups.

\section{DISCUSSION}

In the last few years the Egyptian community demonstrated high rate of tramadol abuse although tramadol has been scheduled. ${ }^{14,15,16}$ The present study evaluates the xerogenic effect of chronic tramadol usage in normal rat model away from its analgesic action.

In the present study, levels of MDA in parotid gland of tramadol treated groups " $20 \& 30$ days" were elevated then reduced after withdrawal of tramadol "20\&30 days" indicating increase in lipid peroxidation during tramadol treatment and its reduction after tramadol withdrawal. Increased levels of MDA were also recorded in chronic heroin users ${ }^{17}$, kidney and liver of rat models of morphine and tramadol treatment. ${ }^{18,19}$ Another model of opioid neurotoxicity found elevated levels of serum MDA in rat model of tramadol and morphine treatment. ${ }^{20}$

In a rat model, parotid protein concentration was enhanced in the fluid response to acetylcholine (1 $\mu \mathrm{mol} / \mathrm{kg}$ ) before and after intravenous tramadol administration $(16 \mu \mathrm{mol} / \mathrm{kg})$ indicating that total protein output is more sensitive to tramadol effect than the fluid response. ${ }^{21}$ This sensitivity of protein could support the results of the current study of toluidine blue stain that showed faintly stained cytoplasm and depleted acini in tramadol group (20\&30 days) after withdrawal of tramadol secretory granules stared to increase in number gradually. Axadalla et al, stated that Nissl granules stained pale blue in the hioppocampaus of rat brain indicating decrease in the Nissl substance "rough endoplasmic reticulum inside the cell bodies and dendrites" after tramadol treatment. ${ }^{22}$

Histological findings of the current study showed disorganization, vacuolization and apoptotic signs in parotid acinar cells, these data are in accordance with other histological findings in brain ${ }^{20,23}$, liver and kidney of rat models of tramadol usage. ${ }^{24}$ Vacuolization in parotid acinar cells could be attributed to cell organoids damage from exposure to free radicals. ${ }^{25,26}$

Among Caspases, Caspase-3 is the key factor in apoptosis, caspase- cascades play an important role in initiation, transduction, and elaboration of apoptotic signals. ${ }^{27}$ Our results showed increased expression of caspase-3 among tramadol treated groups (20\&30 days) and expression was reduced after withdrawal of tramadol. These data were in accordance with the findings of Sharifipour et al, who reported marked intracellular upregulation of caspase-3 in chronic administration of opiate in rats. ${ }^{28}$ Lemarie et al explained that generation of ROS resulted from inhibition of Complex III of the electron transfer chain in mitochondria after high doses of tramadol ${ }^{29}$, which subsequently could induce apoptosis. ${ }^{30}$

Götrick and Tobin stated that the principal xerogenic effect of tramadol was through activating inhibitory pathways in the central nervous system. ${ }^{21}$ According to the current data, we can add that xerogenic effect of tramadol is not only as a result of its action on the nervous system but also due to its oxidative damage and apoptosis of the acinar cells of the parotid gland.

On conclusion, chronic consumption of tramadol induces histological changes in the acinar cells of 
the parotid gland as a result of increased levels of MDA levels and consequently results in apoptosis of the acinar cells. According to the currant data these changes are reversible and the acinar cells almost returned to its normal structure after tramadol withdrawal.

\section{REFERENCES}

1. Garrett JR, Anderson LC. Rat sublingual salivary glands: secretory changes on parasympathetic or sympathetic nerve stimulation and a reappraisal of the adrenergic innervation of striated ducts. Arch Oral Biol 1991: 36: 675- 683.

2. Spence C. Mouth-watering: the influence of environmental and cognitive factors on salivation and gustatory/flavor perception. J Texture Stud 2011: 42: 157-171.

3. Proctor GB. Medication-induced dry mouth. In: Carpenter GH, editor. Dry mouth: a clinical guide on causes, effects and treatments. London: Springer, 2014: 18.

4. Ozyilmaz K, Ayoglu H, Okyay R D, Yurtlu S, Koksal B, Hanci V, Erdogan G, Turan I O. Postoperative analgesic effects of wound infiltration with tramadol and levobupivacaine in lumbar disk surgeries. J Neurosurg Anesthesiol. 2012: 24: 331-335.

5. Lewis KS, Han NH - Tramadol: a new centrally acting analgesic. Am J Health Syst Pharm, 1997; 54: 643- 652.

6. Raffa RB. Basic pharmacology relevant to drug abuse assessment: tramadol as example. J. Clin. Pharm. Ther. 2008: 33(2):101-8.

7. Go"trick B, $\mathrm{A}^{\circ}$ kerman S, Ericson D, Torstenson R, Tobin, G. Oral pilocarpine for treatment of drug-induced oral dryness in healthy adults. J Dent Res, 2004: 83(5):393-397.

8. Loostro"m $\mathrm{H}, \mathrm{A}^{\circ}$ kerman $\mathrm{S}$, Ericson D, Tobin G, Go“ trick B. Tramadol-induced oral dryness and pilocarpine treatment: Effects on total protein and IgA. Arch Oral Biol 2011: $56: 395-400$

9. El-Gaafarawi II. Biochemical toxicity induced by tramadol administration in male rats. Egypt J Hosp Med 2006: 23: 353-362.

10. Pan H Z, Zhang H, Chang D, Li H, Sui H. The change of oxidative stress products in diabetes mellitus and diabetic retinopathy. Br. J. Ophthalmol. 2008: 92(4): 548-551

11. Lushchak V I. 2014. Classification of oxidative stress based on its intensity. EXCLI J. 13: 922-937.
12. Cakatay U. Protein oxidation parameters in type 2 diabetic patients with good and poor glycaemic control. Diabetes Metab. 2005: 31(6): 551-557.

13. Awadalla E, Salah-Eldin AE. Histopathological and molecular studies on tramadol mediated hepato-renal toxicity in rats. IOSR J. Pharm. Biol. Sci. 2015: 10(6): 90-102.

14. Nazarzadeh, M., Bidel, Z., Carson, K.V. The association between tramadol hydrochloride misuse and other substances use in an adolescent population: Phase I of a prospective survey. Addictive Behaviors. 2014: 39: 333-337.

15. Fawzi, M.M. Some medicolegal aspects concerning tramadol abuse: The new Middle East youth plague 2010. An Egyptian overview. Egyptian Journal of Forensic Sciences. 2011: 1: 99-102.

16. Mohamed, N.R., El Hamrawy, L.G., Shalaby, A.S., El Bahy, M.S. and Abd Allah, M.M. An epidemiological study of tramadol $\mathrm{HCl}$ dependence in an outpatient addiction clinic at Heliopolis Psychiatric Hospital. Menoufia Med J. 2015: 28:591-596

17. Panchenko LF, Pirozhkov SV, Nadezhdin AV, Baronets VI, Usmanova NN. Lipid peroxidation, peroxyl radical scavenging system of plasma and liver and heart pathology in adolescence heroin users. Vopr Med Khim 1999: 45: 501-506.

18. Zhang YT, Zheng QS, Pan J, Zheng RL. Oxidative damage of biomolecules in mouse liver induced by morphine and protected by antioxidants. Basic Clin Pharmacol Toxicol 2004: 95: 53-58.

19. Atici S, Cinel I, Cinel L, Doruk N, Eskandari G, Oral U. Liver and kidney toxicity in chronic use of opioids: an experimental long term treatment model. J Biosci 2005; 30:245-252.

20. S. Atici, L. Cinel, I. Cinel, N. Doruk, M. Aktekin, A. Akca, H. Camdeviren, U. Oral, Opioid neurotoxicity: comparison of morphine and tramadol in an experimental rat model, Int. J. Neurosci. 2004: 114 (8) 1001-1011.

21. Bengt Go "trick, Gunnar Tobin. The xerogenic potency and mechanism of action of tramadol inhibition of salivary secretion in rats. Arch Oral Biol (2004) 49, 969-973.

22. Awadalla E, Salah-Eldin A. Molecular and histological changes in cerebral cortex and lung tissues under the effect of tramadol treatment. Biomedicine \& Pharmacotherapy. 2016: 82: 269-280 
23. H. Zhuo, L. Huang, H. Huanga, Z. Cai, Effects of chronic tramadol exposure on the zebrafish brain: a proteomic study, J. Proteom. 2012:75 (11): 3351-3364.

24. Obed Khalid Ali, AL- Jmor Sara Ahmed, Al-Graibawi Mawlood. A. A. Effects of Tramadol on Histopathological and Biochemical Parameters in Male Rabbits. American Journal of Biology and Life Sciences. 2015: 3(3) 85-90.

25. Zarnescu O, Brehar FM, Chivu M, Ciurea AV. Immunohistochemical localization of caspase-3, caspase-9 and Bax in U87 glioblastoma xenografts. J Mol Histol 2008: 39: 561-569.

26. Brown DM, Donaldson K, Borm PJ, Schins RP, Dehnhardt M, Gilmour P, L. A. Jimenez, V. Stone. Calcium and ROSmediated activation of transcription factors and TNFalpha cytokine gene expression in macrophages exposed to ultrafine particles. Am J Physiol Lung Cell Mol Physiol 2004: 286: 344-353.

27. Cohen G.M. Caspases: the executioners of apoptosis, Biochem. J. 1997: 326 1- 16.

28. Sharifipour M, Izadpanah E, Nikkhoo B, Zare S, Abdolmaleki A, Hassanzadeh K, Moradi F, Hassanzadeh K. A new pharmacological role for donepezil: attenuation of morphine-induced tolerance and apoptosis in rat central nervous system. J Biomed Sci 2014; 23: 21-26.

29. Lemarie A, Grimm S. Mutations in the heme b-binding residue ofSDHC inhibit assembly of respiratory chain complex II in mammaliancells. Mitochondrion 2009; 9: 254-260.

30. Chandra J. Free Radic. Biol. Med. 29 (2000) 32. 